\title{
Maraviroc Concentrates in the Cervicovaginal Fluid and Vaginal Tissue of HIV-Negative Women
}

\author{
Julie B. Dumond, PharmD*, Kristine B. Patterson, MD $\dagger$, Allison L. Pecha, PharmD ${ }^{\star}$, Rebecca \\ E. Werner, BS $\dagger$, Emma Andrews, PharmD $\ddagger$, Bharat Damle, PhD $\ddagger$, Randall Tressler, MD $\ddagger$, \\ Jochen Worsley, PhD $¥$, and Angela D. M. Kashuba, BScPharm, PharmD, DABCP* \\ *Division of Pharmacotherapy and Experimental Therapeutics, University of North Carolina at \\ Chapel Hill Eshelman School of Pharmacy, Chapel Hill, NC \\ tDepartment of Medicine, Infectious Diseases Division, University of North Carolina at Chapel Hill \\ School of Medicine, Chapel Hill, NC \\ ‡Pfizer Global Research and Development, New York, NY.
}

\begin{abstract}
Objective-To compare single- and multiple-dose maraviroc exposures in cervicovaginal fluid (CVF) and vaginal tissue (VT) with blood plasma (BP) and quantify maraviroc protein binding in CVF.

Design-Open-label pharmacokinetic study.

Methods-In 12 HIV-negative women, 7 paired CVF and BP samples were collected over 12 hours after 1 maraviroc dose. Subjects then received maraviroc twice daily for 7 days. After the last dose, subjects underwent $\mathrm{CVF}$ and BP sampling as on day 1, with additional sampling during terminal elimination. VT biopsies were obtained at steady state.
\end{abstract}

Results-Day 1 and day 7 median maraviroc $\mathrm{CVF} \mathrm{AUC}_{\tau}$ were 1.9- and 2.7-fold higher, respectively, than BP. On day 1,6 of 12 subjects had detectable maraviroc CVF concentrations within 1 hour; 12 of 12 were detectable within 2 hours, and all exceeded the protein-free $\mathrm{IC}_{90}$. On day 7 , maraviroc CVF protein binding was $7.6 \%$ and the $\mathrm{VT} \mathrm{AUC}_{\tau}$ was 1.9 -fold higher than BP. Maraviroc CVF concentrations 72 hours after dose and BP concentrations 12 hours after dose were similar.

Conclusions-Higher maraviroc exposure in the female genital tract provides a pharmacologic basis for further evaluation of chemokine receptor 5 antagonists in HIV infection prophylaxis. This is the first study to report antiretroviral VT concentrations, CVF protein binding, and CVF terminal elimination.

\section{Keywords}

CCR5 antagonist; cervicovaginal; genital tract; maraviroc; pharmacokinetic; tissue; vaginal

\footnotetext{
Copyright $@ 2009$ by Lippincott Williams \& Wilkins

Correspondence to: Angela D. M. Kashuba, BScPharm, PharmD, DABCP, University of North Carolina at Chapel Hill, Eshelman School of Pharmacy, 3318 Kerr Hall, CB \#7360, Chapel Hill, NC 27599-7360 (akashuba@unc.edu)..

Data presented previously at the 15th Conference on Retroviruses and Opportunistic Infections, February 3-6, 2008, Boston, MA (Abstract 135LB).
} 


\section{INTRODUCTION}

HIV/AIDS continues to be a serious public health concern that demands attention. Although antiretroviral therapy has drastically reduced HIV-associated mortality and improved the quality of life of those living with HIV infection, ${ }^{1-3}$ for every person treated, 4 new people become infected. ${ }^{1}$ Sexual transmission of HIV remains the primary mode of HIV transmission worldwide. ${ }^{1,4}$ Without an effective vaccine, alternative strategies to prevent the sexual transmission of HIV are critical.

There are 3 ways antiretroviral therapy can be used to prevent HIV transmission: pre-exposure prophylaxis (PrEP) (both topically and orally), postexposure prophylaxis (PEP) (orally), and treatment of the infected individual to reduce HIV replication in genital secretions. As females are especially vulnerable to the sexual transmission of HIV, understanding the degree to which antiretrovirals (ARVs) concentrate in the female genital tract is particularly important in both primary and secondary prevention. ${ }^{4-7}$

Discordance in exposure of ARVs between the genital tract and blood plasma (BP) has both individual and public health implications. On an individual level, there is a risk for harboring resistant virus in sanctuary sites such as the genital tract, which in turn can potentially reseed the systemic compartment resulting in treatment failure. From a public health perspective, inadequate penetration of ARVs into the female genital tract may result in ongoing genital shedding, even in the presence of suppressed plasma HIV RNA, thereby increasing the risk of sexual transmission of HIV. ${ }^{8-10}$

Although the exact mechanism of the sexual transmission of HIV-1 within the female genital tract is not fully understood, viruses that utilize the CCR5 chemokine receptor predominate in the early stages of mucosal transmission. ${ }^{5,6,11-14}$ Modeling of a male-to-female transmission has proposed a direct correlation between the number of endocervical CCR5 receptors and the probability of HIV-1 infection. ${ }^{14}$ Therefore, the CCR5 receptor is an appealing target for HIV prophylaxis. Proof of concept has been demonstrated in a nonhuman primate model using a novel oral CCR5 receptor antagonist (CMPD167). After vaginal simian HIV (SHIV) challenge, CMPD167 could both prevent infection and lower the viral set point after infection. ${ }^{15}$

The present study was designed to understand CCR5 antagonist exposure in the human female genital tract. Using the commercially available CCR5 antagonist maraviroc (Celsentri/ Selzentry; Pfizer, Inc, New York, NY), single- and multiple-dose exposures in cervicovaginal fluid (CVF), BP, and vaginal tissue (VT) were investigated.

\section{METHODS}

\section{Study Design and Subject Selection}

This 10-day, open-label, pharmacokinetic (PK) study in healthy HIV-negative female volunteers was conducted between April 2007 and June 2007 at the University of North Carolina at Chapel Hill (UNC). All subjects provided written informed consent, including a separate consent for HIV testing. The protocol was approved by the UNC Biomedical Institutional Review Board.

Subjects were eligible to participate if they were healthy premenopausal females with an intact uterus and cervix and body mass index between 18 and $30 \mathrm{~kg} / \mathrm{m}^{2}$ with a total body weight $\geq 50$ $\mathrm{kg}$. Subjects were required to be between the ages of 18 and 49 years and using an acceptable form of birth control. Subjects were excluded if they were pregnant or nursing, had a history of regular alcohol consumption, or currently smoking more than 5 cigarettes per day. Subjects with a history of loop electrosurgical excision procedure, conization, or cryosurgery were 
excluded to minimize any potential variability in sample collection. Subjects were excluded for a positive urine drug screen; presence of a sexually transmitted infection; or infection with HIV-1, hepatitis B, or hepatitis C. Subjects were excluded for any abnormal screening laboratory results or any clinically significant abnormality on physical and/or pelvic examination deemed by the study safety officer to increase subject risk or compromise study results. Subjects were excluded from study participation if they exhibited signs and symptoms of orthostasis or a QTc $>450$ milliseconds. Twelve-lead electrocardiogram (EKG) testing was performed per standard Pfizer protocol for healthy volunteer studies. Subjects were permitted to use oral hormonal contraceptives and up to $1 \mathrm{~g} / \mathrm{d}$ of acetaminophen. All other medications were discontinued at least 7 days before study drug dosing and could not be restarted until after study completion. Subjects were instructed to abstain from sexual intercourse and the use of vaginal products from 48 hours before dosing until study discharge.

Screening procedures occurred within the 28 days before maraviroc dosing. Comprehensive laboratory studies, including a complete blood count with differential, liver function tests, serum chemistries, hepatitis B and C serology panels, urinalysis, and serum pregnancy testing, and sexually transmitted infection evaluations, were performed at the screening visit. Sexually transmitted infection evaluations included testing for gonorrhea, chlamydia, trichomoniasis (Aptima Combo 2 and Aptima TV ASR Kits; Gen-Probe Incorporated, San Diego, CA), bacterial vaginosis (QuickVue Advance $\mathrm{pH}$ and amine kit; Quidel Corp, San Diego, CA), and syphilis. Hepatitis simplex virus-2 antibody (Focus Diagnostics, Cypress, CA) was measured if indicated by the presence of suspected herpetic lesions on examination. All subjects underwent anonymous HIV testing using a standard HIV-1/2 enzyme-linked immunosorbent assay with pooled HIV-1 polymerase chain reaction testing. ${ }^{16}$ All testing was performed in the McLendon Laboratories of UNC Hospitals or in the UNC Sexually Transmitted Diseases Cooperative Research Center Microbiology Core Lab.

Safety laboratory monitoring was performed on days $0,4,8$, and follow-up. A full physical examination was performed at screening and at follow-up, and brief physical examinations were performed on days 0 and 8 . Single supine and standing blood pressure and pulse measurements and a single 12-lead EKG were obtained on day 0, day 8, and at follow-up.

\section{Study Visits}

During the treatment phase of the study, subjects received maraviroc $300 \mathrm{mg}$ orally twice daily on days 1-6 and a single 300-mg dose in the morning of day 7. To obtain maximal CVF volume, day 1 was scheduled to occur within 7-10 days after the end of the subject's menses. Subjects were admitted the evening before day 1 to the UNC Verne S. Caviness General Clinical Research Center (CRC) for intensive PK sampling. Subjects fasted for 8 hours before dosing and 4 hours after dosing on days 1 and 7 and refrained from drinking water for at least 1 hour before and after the maraviroc dose. In the morning of day 1, paired BP and CVF samples were obtained at pre-dose, 1, 2, 4, 6, 8, and 12 hours after first dose. Standard meals were provided to subjects at the appropriate times. Subjects returned to the CRC each morning on days 2-6 for trough BP and CVF PK sampling before the morning dose of study medication. Subjects were instructed to fast for 2 hours before and 1 hour after the morning dose and to restrict water intake for 1 hour before and after dose. Morning doses were witnessed, and subjects were given the evening dose to take home with a diary to record the time of dosing. Subjects were directed to follow the same dosing instructions in the evening. Subjects were readmitted to the CRC in the evening of day 6 . In the morning of day 7, intensive BP and CVF PK sampling identical to day 1 was performed. A single VT biopsy was obtained at one of the following time points after dose: 2, 4, 8, and 12 hours. Subjects remained in the CRC until the morning of day 8 . At this time, a 24-hour post-dose BP and CVF PK sample was obtained and safety studies were conducted. All subjects returned to the $\mathrm{CRC}$ in the mornings of days 9 and 10 for $\mathrm{BP}$ and $\mathrm{CVF}$ 
PK sampling 48 and 72 hours after the final dose. Subjects returned for a safety follow-up visit 7-10 days after the last dose of maraviroc.

\section{Sample Collection and Processing}

CVF samples were self-collected via direct aspiration with a volumetric aspiration device. ${ }^{17}$, ${ }^{18}$ After collection, CVF was transferred to labeled cryovials and stored at $-80^{\circ} \mathrm{C}$ until analysis. Whole blood was obtained using sodium heparin-containing collection tubes (BD Diagnostics, Franklin Lakes, NJ) and centrifuged at $1300 \mathrm{~g}$ at $4^{\circ} \mathrm{C}$ for 10 minutes. The resulting $\mathrm{BP}$ was aliquoted into labeled cryovials and stored at $-80^{\circ} \mathrm{C}$ until analysis.

VT was collected by biopsy. Briefly, subjects were placed in the dorsal lithotomy position, and $\mathrm{CVF}$ was collected with the aspirator by the clinician before speculum insertion. After speculum insertion, the right or left vaginal fornix was cleaned with povidone-iodine, the anticipated biopsy site was anesthetized with topical $20 \%$ benzocaine spray (HurriCaine; Beutlich Pharmaceuticals, Waukegan, IL), and an approximately $3 \times 3 \times 1$-mm specimen was obtained using Baby Tischler forceps (Cooper Surgical, Trumbull, CT). Samples were placed in a labeled screw-capped polypropylene tube, immediately snap frozen in liquid nitrogen, and stored at $-80^{\circ} \mathrm{C}$ until analysis.

Protein precipitation was used to prepare $\mathrm{BP}, \mathrm{CVF}$, and tissue samples for analysis. Briefly, to $50 \mu \mathrm{L}$ of $\mathrm{BP}, \mathrm{CVF}$, or VT homogenate, $700 \mu \mathrm{L}$ of an acetonitrile solution containing $1 \mathrm{mg} /$ $\mathrm{mL}$ of the internal standard maraviroc- $\mathrm{d}_{5}$ was added. After vortex and centrifugation, $400 \mu \mathrm{L}$ of supernatant was transferred to a clean tube and tightly sealed for analysis.

After maraviroc concentration analysis, remaining day 7 samples of CVF were pooled for equilibrium dialysis to determine protein binding. Pooling of samples was necessary due to the volume (many samples $<500 \mu \mathrm{L}$ ) of CVF collected at each time point. Briefly, $200 \mu \mathrm{L}$ of pooled CVF sample was incubated at $37^{\circ} \mathrm{C}$ for 16 hours in rapid equilibrium dialysis cartridges (Pierce Biotechnology, Inc, Rockford, IL). Subsequently, $50 \mu \mathrm{L}$ from the rapid equilibrium dialysis well was transferred to 1 well of a 96-well plate, and $50 \mu \mathrm{L}$ of phosphate-buffered saline added. Similarly, $50 \mu \mathrm{L}$ from the phosphate-buffered saline well was transferred to 1 well of a 96-well plate, and $50 \mu \mathrm{L}$ of "blank" vaginal fluid added. These samples were treated by the protein precipitation method described above before analysis.

Maraviroc concentrations for all samples were analyzed using validated high-performance liquid chromatography-tandem mass spectrometry methods. All analyses were performed using a LEAP autosampler (LEAP Technologies, Chapel Hill, NC), a Rheos 2000 liquid chromatograph (Flux Instruments AG, Basel, Switzerland), a Fluophase PFP column (4.6 × $50 \mathrm{~mm}, 5 \mu \mathrm{m}$; Thermo Electron Corporation, Pittsburgh, PA), and an API 4000 (MDS Sciex, Thornhill, Ontario). The API 4000 instrument was used in positive TurboIonSpray mode, with a source temperature of $550^{\circ} \mathrm{C}$. The mobile phase consisted of 80:20 vol:vol acetonitrile: 25 $\mathrm{mM}$ ammonium acetate in aqueous $0.2 \%$ formic acid, at a flow rate of $1 \mu \mathrm{L} / \mathrm{min}$. Analytes were detected using multiple reaction monitoring, and the precursor/product ion transitions were $\mathrm{m} /$ $z 514.1 \rightarrow 389.1$ for maraviroc and $\mathrm{m} / z, 519.1 \rightarrow 394.1$ for maraviroc- $\mathrm{d}_{5}$. The dynamic range of the assay was $0.5-500 \mathrm{ng} / \mathrm{mL}$ for BP and CVF and 20-20,000 ng/g for VT.

Validations for BP, CVF, VT, and protein binding demonstrated maraviroc recoveries of $\geq 80 \%$ for all matrices. Accuracy and imprecision experiments demonstrated that for the lower limit of quantification (LLQ), the overall mean concentration deviated $\leq 20.0 \%$ from target, with relative standard deviation (RSD) $\leq 20.0 \%$. For the low, medium, and high controls, overall mean concentration deviated $\leq 15.0 \%$ from target, with an RSD $\leq 15.0 \%$ 


\section{Data Analysis}

Subject demographic information was collected and summarized descriptively. BP and CVF PK parameters were estimated using noncompartmental methods. The maximum concentration $\left(C_{\max }\right)$ was estimated directly from experimental data; $T_{\max }$ was defined as the time of first occurrence of $C_{\max }$. The area under the plasma concentration-time curve (AUC) within the dosing interval $(\tau)$, where $\tau=12$ hours for maraviroc, was estimated using the log-linear trapezoidal method. To compare exposure in the genital tract with that of BP, CVF:BP $\mathrm{AUC}_{\tau}$ ratios were calculated for days 1 and 7 . To describe multidose accumulation in $\mathrm{BP}$ and $\mathrm{CVF}$, day 7:day $1 \mathrm{AUC}_{\tau}$ ratios were calculated. $\mathrm{BP}$ and $\mathrm{CVF} C_{12 \mathrm{~h}}\left(C_{\min }\right)$ concentrations collected on days 2-6 were plotted per subject and visually inspected to confirm steady state conditions on day 7 .

To perform a concentration comparison between VT and CVF and BP, an estimated tissue density of $1.05 \mathrm{~g} / \mathrm{mL}$ was used to convert nanograms per gram to nanograms per milli-liter. To estimate VT PK parameters, a composite approach was used: At each time point, the median VT concentration was calculated and the composite concentration-time profile generated. To compare median exposure in VT samples relative to $\mathrm{BP}$, this composite $\mathrm{AUC}_{\tau}$ was divided by

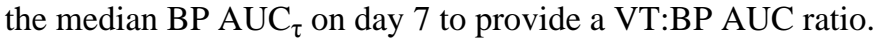

Descriptive statistics were generated by SAS Institute, Inc (Cary, NC), software version 8.2. Data are presented as median (interquartile range), unless otherwise specified.

\section{RESULTS}

\section{Subject Demographics, Disposition, and Safety}

Of 21 women screened for this study, 13 were enrolled in the protocol and 12 provided PK samples. One subject was withdrawn by investigators due to active vaginal bleeding at the first study visit, related to a missed oral contraceptive tablet earlier in the week of the scheduled visit; maraviroc was not administered, and this subject did not contribute any data. Demographic information is presented in Table 1 . The mean age of the 12 participants was 26.9 years, mean weight was $63.0 \mathrm{~kg}$, and the mean body mass index was $22.2 \mathrm{~kg} / \mathrm{m}^{2}$. Six of 12 subjects were white. Nine of 12 women received concomitant oral contraceptive therapy.

Of these 12 subjects, 1 withdrew from the protocol for personal reasons before the day 7 PK visit. An additional subject did not complete maraviroc blood sampling or vaginal biopsy on day 7 but did complete day $7 \mathrm{CVF}$ collections due to a vasovagal response at the 4 hours postdose blood draw. The study physician/safety officer deemed this vasovagal reaction to be unrelated to study medication, as this event occurred in relation to obtaining a BP sample; the subject was supine at the time of the episode, and the subject reported similar reactions during phlebotomy in the past. She recovered fully before discharge on day 8 . The remaining 10 subjects completed the protocol as planned.

Subjects tolerated the study medication well. The majority of adverse events (AEs) were mild in severity, and there were no serious AEs reported. The most frequently reported AEs were fatigue (25\%), nausea (17\%), and headache (17\%), similar to previous reports. Eosinophilia, tinnitus, vomiting, abdominal pain, flatulence, and somnolence were reported by at least 1 subject and attributed to maraviroc. No EKG abnormalities were related to maraviroc administration. Subjects also tolerated CVF and VT sampling well; post-biopsy vaginal bleeding was minimal and resolved within $24-48$ hours of the procedure. 


\section{BP and CVF Pharmacokinetics}

Figures $1 \mathrm{~A}$ and $\mathrm{B}$ depict the median (interquartile range) $\mathrm{BP}$ and CVF concentrations for all subjects at day $1(n=12)$ and day $7(n=10)$, respectively. One hour after the first dose of maraviroc (the earliest sampling time point), BP concentrations were detectable in 12 of 12 women and CVF concentrations were detectable in 6 of 12 women. By 2 hours, 12 of 12 women had detectable maraviroc in their CVF. Median CVF concentrations surpassed BP concentrations by 4 hours and remained higher than BP for the duration of the dosing interval. On day 7, median CVF concentrations were consistently higher than BP after the 4-hour time point.

Figure $1 \mathrm{C}$ shows $\mathrm{BP}$ and $\mathrm{CVF}$ trough concentrations $\left(C_{\min }\right)$ obtained immediately before dosing on days 2-6. Blood draws on days 2-6 were occasionally omitted due to concerns over multiple needle insertions, limiting the ability to obtain venous access for PK sampling on day 7. This is reflected in $11 \mathrm{BP}$ samples obtained on day 3 and in only $6 \mathrm{BP}$ samples obtained on day 5. However, all subjects provided all scheduled CVF samples. All samples collected had detectable maraviroc concentrations, and CVF trough concentrations were consistently higher than those of BP. These data also confirmed that steady state conditions were achieved by day 7.

Table 2 summarizes the $\mathrm{PK}$ parameters for each matrix, along with accumulation ratios (day 7:day 1) and CVF:BP ratios. Maraviroc BP parameters were consistent with data from historical controls. ${ }^{19,20}$ Time to maximal concentration $\left(T_{\max }\right)$ at day 7 was delayed 3 hours in CVF compared with $\mathrm{BP}$, and maximal concentrations $\left(C_{\max }\right)$ in $\mathrm{CVF}$ at day 7 were 2.2-fold higher than in BP. Maraviroc moderately accumulated in BP over 7 days of dosing by $26 \%$ [day 7:day $1 \mathrm{AUC}_{\tau}$ ratio $\left.=1.26(1.16-1.30)\right]$. However, maraviroc significantly accumulated in CVF by $213 \%$ [day 7:day $1 \mathrm{AUC}_{\tau}$ ratio $=2.13(1.16-2.59)$ ]. When CVF exposure was compared with $\mathrm{BP}$ exposure, the CVF:BP $\mathrm{AUC}_{\tau}$ ratio on day 1 was $1.91(0.82-4.77)$ and on day 7 was 2.73 (1.0-5.39). This demonstrates that CVF exposures were approximately 2 -fold higher than BP on day 1 and 2.7-fold higher than BP on day 7. Individual CVF:BP AUC ratios for days 1 and 7 are shown in Figure 1D. No correlation was found between BP exposure and CVF exposure either on day 1 or on day 7 of dosing.

Single VT samples were collected from 10 subjects; 3 subjects' samples were collected at each of the 2- and 4-hour post-dose time points, and 2 subjects' samples were collected at each of the 8- and 12-hour post-dose time points. Tissue concentrations were measured in nanograms per gram and corrected for tissue density $(1.05 \mathrm{~g} / \mathrm{mL})$ to determine an equivalent concentration in nanograms per milliliter. The median VT concentrations are shown in Figure 2A, along with the comparative BP and CVF median concentrations over the dosing interval on day 7. VT composite PK parameter estimates are provided in Table 2. Maraviroc VT exposure was 1.9fold higher than BP exposure and approximately $65 \%$ of the CVF exposure (VT:CVF $\mathrm{AUC}_{\tau}$ ratio $=0.65)$.

After 7 days of dosing, the terminal elimination of maraviroc in BP and CVF was evaluated by sampling 24, 48, and 72 hours after the final dose (Fig. 2B). CVF concentrations exhibited a linear and predictable decline and paralleled that of BP. CVF concentrations were approximately 10 times higher than $\mathrm{BP}$ at the end of the 12-hour dosing interval, and CVF concentrations at 72 hours after dose were similar to BP concentrations 12 hours after dosing.

Protein binding was performed on pooled CVF samples obtained during day 7 of maraviroc dosing. Ten samples at, or close to, $C_{\max }$ were pooled into 4 samples for analyses. In these samples, protein binding ranged from $3.7 \%$ to $13.6 \%$ with a mean of $7.6 \%$. 


\section{DISCUSSION}

This study investigated the female genital tract exposure of maraviroc, the first commercially available CCR5 antagonist. This comprehensive evaluation also included, for the first time, measurements of VT concentrations, CVF protein binding, and terminal elimination of drug in cervicovaginal secretions. Maraviroc achieved one of the highest CVF exposures of 17 ARVs evaluated to date. ${ }^{17,18,21,22}$ Based on the physicochemical properties of maraviroc, in addition to its protein binding affinity, these results were unexpected. CVF exposures exceeded those of plasma by a median of 1.9-fold and 2.7-fold after single and multiple dosing, respectively. Additionally, maraviroc CVF concentrations were observed in $50 \%$ of the women 1 hour after dosing and in 100\% of the women 2 hours after dosing. VT exposures were 1.9fold higher than plasma at steady state. All concentrations were well above the protein-free $\mathrm{IC}_{90}$ of $0.5 \mathrm{ng} / \mathrm{mL} .{ }^{23}$

The mechanism for these increased GT concentrations is not known but could be related to drug transport or altered drug metabolism in genital tract tissue. Little is known about the drug disposition properties of the female GT, although P-glycoprotein (an efflux transporter) has been found in several female GT tissues. ${ }^{24}$ Additionally, compared with systemic drug exposure, disproportionately increased seminal concentrations of the protease inhibitor indinavir in the presence of ritonavir (a potent inhibitor of P-glycoprotein) have been described, 25 providing support for the potential role of drug transporters in GT drug disposition. However, no such data exist in women. Elucidating the determinants of drug penetration in the GT has important implications for PrEP/PEP, if drug properties or physiologic mechanisms can be manipulated to improve ARV exposure.

Although this study did not measure maraviroc's affinity for CCR5 receptors on cervical mononuclear cells, receptor occupancy has not been shown to correlate with efficacy in HIVinfected subjects. ${ }^{26}$ However, protein-free concentrations $\geq 0.5 \mathrm{ng} / \mathrm{mL}$ have been demonstrated effective against a wide variety of HIV isolates in cultured donor peripheral blood mononuclear cells. ${ }^{23}$ Median protein-free maraviroc CVF concentrations were 10 -fold higher than this value 1 hour after a single dose and were $>800$-fold higher than this value at $C_{\min }$ under steady state conditions. Therefore, virologic efficacy in this compartment is expected.

Protein binding in the female genital tract was determined for the first time in this study. Maraviroc protein binding in CVF was approximately 10-fold lower than BP, suggesting that the majority of drug present in the genital tract is unbound and available for pharmacological activity. By achieving higher concentrations in the CVF, maraviroc and other ARVs may be beneficial to HIV-infected women by preventing continued genital tract shedding of HIV, thereby decreasing the risk of secondary transmission to sexual partners. ${ }^{27-29}$ Also, a decrease in genital tract HIV RNA may prevent the emergence of resistant virus within sanctuary sites such as the genital tract, which may reseed the systemic compartment resulting in treatment failure. Although evidence suggests that maraviroc plasma concentrations in HIV-infected patients and healthy volunteers are similar, ${ }^{19}$ further studies investigating genital tract exposure and treatment responses of CCR5 inhibitors within the genital tract in HIV-infected women are warranted.

This study is the first to measure total VT concentrations of an ARV. These data are critical to understanding the pharmacology of maraviroc in the female genital tract, as it is the tissue, rather than the CVF, that is the site of HIV infection. It would seem likely that drugs achieving high exposures in CVF would have correspondingly high concentrations in VT and, likewise, those achieving low CVF exposures with corresponding low VT concentrations. However, without a complete understanding of drug transport and metabolism in the female genital tract, it is premature to extrapolate these results to other ARVs. Although protein-free maraviroc 
tissue concentrations were not determined in this investigation, and biopsy logistics precluded constructing full tissue PK curves for individual subjects, these data are an important first step in understanding ARV drug disposition in female genital tract tissues. Understanding protein binding in female GT tissues will be an important future goal for drugs used in PrEP/PEP.

CCR5 inhibitors may be particularly useful in preventing HIV transmission because viruses that utilize CCR5 predominate in the early stages of mucosal transmission. ${ }^{5-7,12-15}$ Additionally, women with genital ulcer disease demonstrate increased CCR5 receptor concentrations ${ }^{30}$ and are at an increased risk of contracting HIV via heterosexual contact. Thus, the use of a CCR5 inhibitor in this high-risk group of patients may be particularly beneficial. 30-32 Maraviroc quickly achieved concentrations above the protein-free $\mathrm{IC}_{90}$ within 2 hours of administration, suggesting a role in PEP. As maraviroc remained at high concentrations in the genital tract longer than BP after dosing was suspended, this property may allow for more convenient episodic dosing in the prevention arena. The female genital tract exposures of other CCR5 inhibitors in development are currently unknown.

Although pharmacological prophylaxis may be an effective tool in the prevention of heterosexual HIV transmission, its use must balance the risks and benefits for each patient. Recent literature has shown promise regarding the limitations associated with both PrEP and PEP including patient compliance, ${ }^{33-35}$ cost,${ }^{36}$ the development of ARV resistance, ${ }^{37,38}$ and toxicities ${ }^{39,40}$; however, these limitations still exist and must be considered in choosing a PrEP/ PEP regimen. Furthermore, these limitations stress the importance of continued adequate patient counseling, education, and the consistent use of safe sex practices.

Following these promising pharmacologic results, several important areas of investigation remain. These include quantifying the effects of maraviroc on genital tract HIV RNA and sexual infectivity in HIV-infected patients; determining the potential for CCR5 inhibition in the genital tract to select for transmission of dual-mixed tropic or X4-tropic virus; and the impact of chronic CCR5 inhibition in areas with endemic tick-borne illnesses ${ }^{41}$ and West Nile virus. ${ }^{42}$ Nonetheless, with a novel mechanism of action, excellent oral bioavailability, and high genital tract exposure, maraviroc is an attractive candidate for further investigation in the prevention of heterosexual HIV transmission.

\section{ACKNOWLEDGMENTS}

The authors wish to acknowledge Kim Boggess, MD, Gretchen Stuart, MD, and Kimberly Hamden, RN, for technical assistance in performing vaginal biopsies.

Supported by National Institute of Allergy and Infectious Disease/National Institutes of Health (AI54980, ADMK; AI77355, KBP), Building Interdisciplinary Careers in Women's Health (HD001441, KBP), University of North Carolina at Chapel Hill (UNC) Center for AIDS Research (AI50410), UNC General Clinical Research Center (RR00046), and Pfizer Global Research and Development.

\section{REFERENCES}

1. UNAIDS/WHO. AIDS epidemic update.. AIDS Epidemic Update. 2007 [December 1, 2007]. Available online at: http://data.unaids.org/pub/EPISlides/2007/2007_epiupdate_en.pdf.

2. Mocroft A, Ledergerber B, Katlama C, et al. Decline in the AIDS and death rates in the EuroSIDA study: an observational study. Lancet 2003;362:22-29. [PubMed: 12853195]

3. Palella FJ Jr, Delaney KM, Moorman AC, et al. Declining morbidity and mortality among patients with advanced human immunodeficiency virus infection. HIV Outpatient Study Investigators. N Engl J Med 1998;338:853-860. [PubMed: 9516219]

4. National Institutes of Health: National Institute of Allergy and Infectious Disease. HIV infection in women.. In: Anderson, JR., editor. AIDSInfo. Department of Health and Human Services; Rockville: 2006. 
5. Yeaman GR, Asin S, Weldon S, et al. Chemokine receptor expression in the human ectocervix: implications for infection by the human immunodeficiency virus-type I. Immunology 2004;113:524533. [PubMed: 15554931]

6. Hladik F, Sakchalathorn $\mathrm{P}$, Ballweber $\mathrm{L}$, et al. Initial events in establishing vaginal entry and infection by human immunodeficiency virus type-1. Immunity 2007;26:257-270. [PubMed: 17306567]

7. Connor RI, Sheridan KE, Ceradini D, et al. Change in coreceptor use coreceptor use correlates with disease progression in HIV-1-infected individuals. J Exp Med 1997;185:621-628. [PubMed: 9034141]

8. Blankson JN, Persaud D, Siliciano RF. The challenge of viral reservoirs in HIV-1 infection. Annu Rev Med 2002;53:557-593. [PubMed: 11818490]

9. Pierson T, McArthur J, Siliciano RF. Reservoirs for HIV-1: mechanisms for viral persistence in the presence of antiviral immune responses and antiretroviral therapy. Annu Rev Immunol 2000;18:665708. [PubMed: 10837072]

10. Reddy YS, Kashuba A, Gerber J, et al. Roundtable report: importance of antiretroviral drug concentrations in sanctuary sites and viral reservoirs. AIDS Res Hum Retroviruses 2003;19:167176. [PubMed: 12689408]

11. Veazey RS, Klasse PJ, Ketas TJ, et al. Use of a small molecule CCR5 inhibitor in macaques to treat simian immunodeficiency virus infection or prevent simian-human immunodeficiency virus infection. J Exp Med 2003;198:1551-1562. [PubMed: 14623909]

12. Dragic T, Litwin V, Allaway GP, et al. HIV-1 entry into CD4+ cells is mediated by the chemokine receptor CC-CKR-5. Nature 1996;381:667-673. [PubMed: 8649512]

13. Patterson BK, Landay A, Andersson J, et al. Repertoire of chemokine receptor expression in the female genital tract: implications for human immunodeficiency virus transmission. Am J Pathol 1998;153:481-490. [PubMed: 9708808]

14. Chakraborty H, Sen PK, Helms RW, et al. Viral burden in genital secretions determines male-tofemale sexual transmission of HIV-1: a probabilistic empiric model. AIDS 2001;15:621-627. [PubMed: 11317000]

15. Veazey RS, Springer MS, Marx PA, et al. Protection of macaques from vaginal SHIV challenge by an orally delivered CCR5 inhibitor. Nat Med 2005;11:1293-1294. [PubMed: 16273102]

16. Pilcher CD, McPherson JT, Leone PA, et al. Real-time, universal screening for acute HIV infection in a routine HIV counseling and testing population. JAMA 2002;288:216-221. [PubMed: 12095386]

17. Dumond JB, Yeh RF, Patterson KB, et al. Antiretroviral drug exposure in the female genital tract: implications for oral pre- and post-exposure prophylaxis. AIDS 2007;21:1899-1907. [PubMed: 17721097]

18. Min SS, Corbett AH, Rezk N, et al. Protease inhibitor and nonnucleoside reverse transcriptase inhibitor concentrations in the genital tract of HIV-1-infected women. J Acquir Immune Defic Syndr 2004;37:1577-1580. [PubMed: 15577412]

19. Selzentry ${ }^{\mathrm{TM}}$ Full Prescribing Information [package insert]. Pfizer Global Research and Development; New York, NY: 2007.

20. Abel, S.; Van der Ryst, E.; Muirhead, G., et al. Pharmacokinetics of single and multiple oral doses of UK-427,857 - a novel CCR5 antagonist in healthy volunteers [abstract no. 547].. Presented at: Conference on Retroviruses and Opportunistic Infections; Boston, MA. February 10-14, 2003;

21. Cohen MS, Gay C, Kashuba AD, et al. Narrative review: antiretroviral therapy to prevent the sexual transmission of HIV-1. Ann Intern Med 2007;146:591-601. [PubMed: 17438318]

22. Solas C, Lafeuillade A, Halfon P, et al. Discrepancies between protease inhibitor concentrations and viral load in reservoirs and sanctuary sites in human immunodeficiency virus-infected patients. Antimicrob Agents Chemother 2003;47:238-243. [PubMed: 12499197]

23. Dorr P, Westby M, Dobbs S, et al. Maraviroc (UK-427,857), a potent, orally bioavailable, and selective small-molecule inhibitor of chemokine receptor CCR5 with broad-spectrum anti-human immunodeficiency virus type 1 activity. Antimicrob Agents Chemother 2005;49:4721-4732. [PubMed: 16251317]

24. Finstad CL, Saigo PE, Rubin SC, et al. Immunohistochemical localization of P-glycoprotein in adult human ovary and female genital tract of patients with benign gynecological conditions. J Histochem Cytochem 1990;38:1677-1681. [PubMed: 1976674] 
25. van Praag RM, Weverling GJ, Portegies P, et al. Enhanced penetration of indinavir in cerebrospinal fluid and semen after the addition of low-dose ritonavir. AIDS 2000;14:1187-1194. [PubMed: 10894283]

26. Rosario MC, Jacqmin P, Dorr P, et al. Population pharmacokinetic/pharmacodynamic analysis of CCR5 receptor occupancy by maraviroc in healthy subjects and HIV-positive patients. Br J Clin Pharmacol 2008;65(Suppl 1):86-94. [PubMed: 18333870]

27. Montaner JS, Hogg R, Wood E, et al. The case for expanding access to highly active antiretroviral therapy to curb the growth of the HIV epidemic. Lancet 2006;368:531-536. [PubMed: 16890841]

28. Quinn TC, Wawer MJ, Sewankambo N, et al. Viral load and heterosexual transmission of human immunodeficiency virus type 1. Rakai Project Study Group. N Engl J Med 2000;342:921-929. [PubMed: 10738050]

29. Castilla J, Del Romero J, Hernando V, et al. Effectiveness of highly active antiretroviral therapy in reducing heterosexual transmission of HIV. J Acquir Immune Defic Syndr 2005;40:96-101. [PubMed: 16123689]

30. Sheffield JS, Wendel GD Jr, McIntire DD, et al. Effect of genital ulcer disease on HIV-1 coreceptor expression in the female genital tract. J Infect Dis 2007;196:1509-1516. [PubMed: 18008231]

31. Sardana K, Sehgal VN. Genital ulcer disease and human immunodeficiency virus: a focus. Int J Dermatol 2005;44:391-405. [PubMed: 15869537]

32. Johnson AM, Laga M. Heterosexual transmission of HIV. AIDS 1988;2(Suppl 1):S49-S56. [PubMed: 3147680]

33. Kahn JO, Martin JN, Roland ME, et al. Feasibility of postexposure prophylaxis (PEP) against human immunodeficiency virus infection after sexual or injection drug use exposure: the San Francisco PEP Study. J Infect Dis 2001;183:707-714. [PubMed: 11181146]

34. Martin JN, Roland ME, Neilands TB, et al. Use of postexposure prophylaxis against HIV infection following sexual exposure does not lead to increases in high-risk behavior. AIDS 2004;18:787-792. [PubMed: 15075514]

35. Cordes C, Moll A, Kuecherer C, et al. HIV transmission despite HIV post-exposure prophylaxis after non-occupational exposure. AIDS 2004;18:582-584. [PubMed: 15090822]

36. Abbas UL, Anderson RM, Mellors JW. Potential impact of antiretroviral therapy on HIV-1 transmission and AIDS mortality in resource-limited settings. J Acquir Immune Defic Syndr 2006;41:632-641. [PubMed: 16652038]

37. Si-Mohamed A, Kazatchkine MD, Heard I, et al. Selection of drug-resistant variants in the female genital tract of human immunodeficiency virus type 1-infected women receiving antiretroviral therapy. J Infect Dis 2000;182:112-122. [PubMed: 10882588]

38. Eron JJ, Vernazza PL, Johnston DM, et al. Resistance of HIV-1 to antiretroviral agents in blood and seminal plasma: implications for transmission. AIDS 1998;12:F181-F189. [PubMed: 9814860]

39. Bassett IV, Freedberg KA, Walensky RP. Two drugs or three? Balancing efficacy, toxicity, and resistance in postexposure prophylaxis for occupational exposure to HIV. Clin Infect Dis 2004;39:395-401. [PubMed: 15307008]

40. Quirino T, Niero F, Ricci E, et al. HAART tolerability: post-exposure prophylaxis in healthcare workers versus treatment in HIV-infected patients. Antivir Ther 2000;5:195-197. [PubMed: 11075939]

41. Kindberg E, Mickiene A, Ax C, et al. A deletion in the chemokine receptor 5 (CCR5) gene is associated with tickborne encephalitis. J Infect Dis 2008;197:266-269. [PubMed: 18179389]

42. Lim JK, Louie CY, Glaser C, et al. Genetic deficiency of chemokine receptor CCR5 is a strong risk factor for symptomatic West Nile virus infection: a meta-analysis of 4 cohorts in the US epidemic. J Infect Dis 2008;197:262-265. [PubMed: 18179388] 

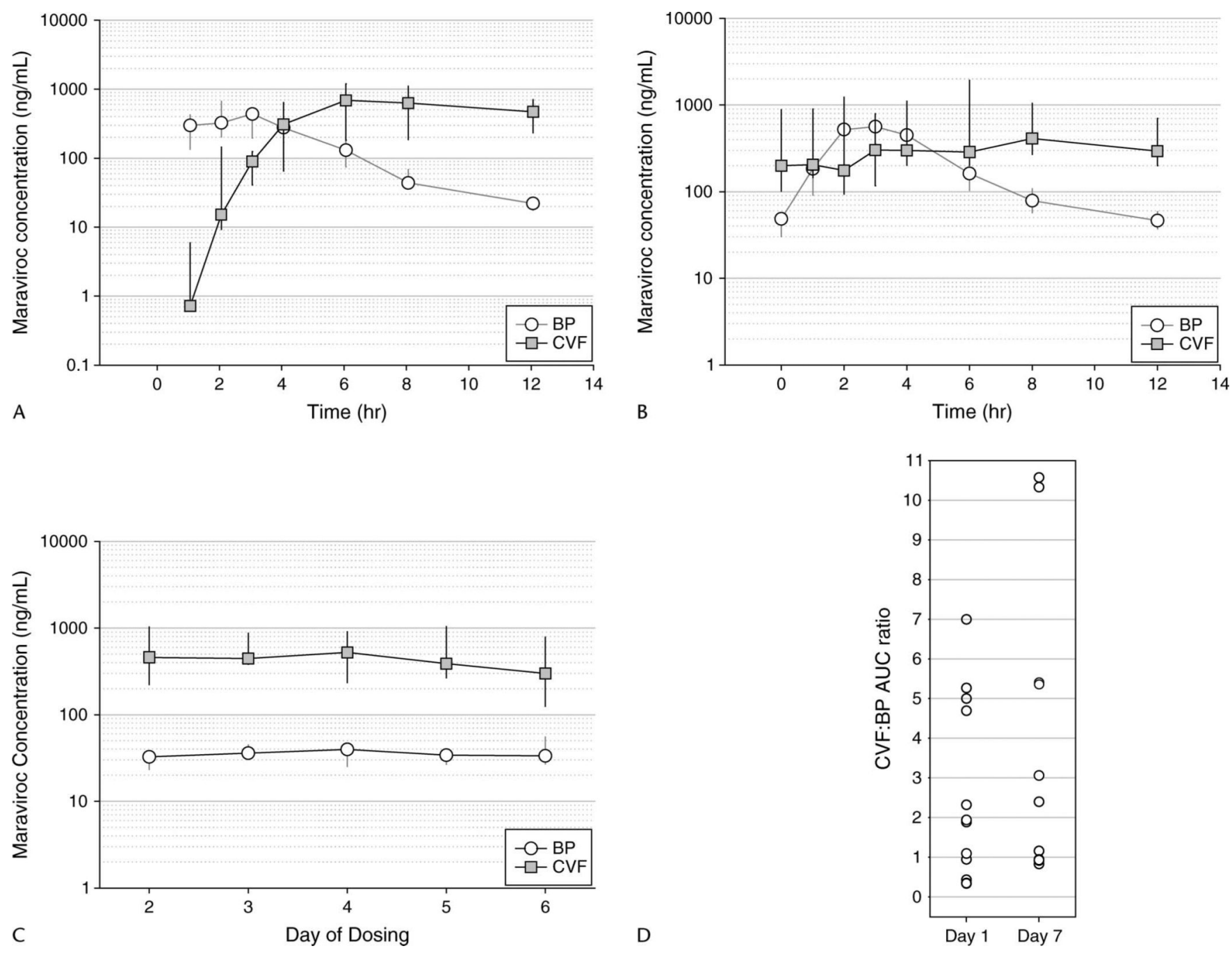

FIGURE 1.

A, Day 1 (single dose) concentration-time profile of maraviroc in BP and CVF for 12 subjects over the 12-hour dosing interval [data presented as median (IQR)]. B, Day 7 (multiple dose) concentration-time profile of maraviroc in BP and CVF for 10 subjects over the 12-hour dosing interval [data presented as median (IQR)]. C, Trough $\left(C_{\min }\right)$ concentration of maraviroc in BP and CVF over days 2-6 of dosing [data presented as median (IQR)]. For all CVF time points, $\mathrm{n}=12$; for BP, day 3 included 11 subjects and day 5 included 6 subjects. D, Individual CVF:BP AUC ratios calculated over a day 1 and day 7 dosing interval. IQR, interquartile range. 

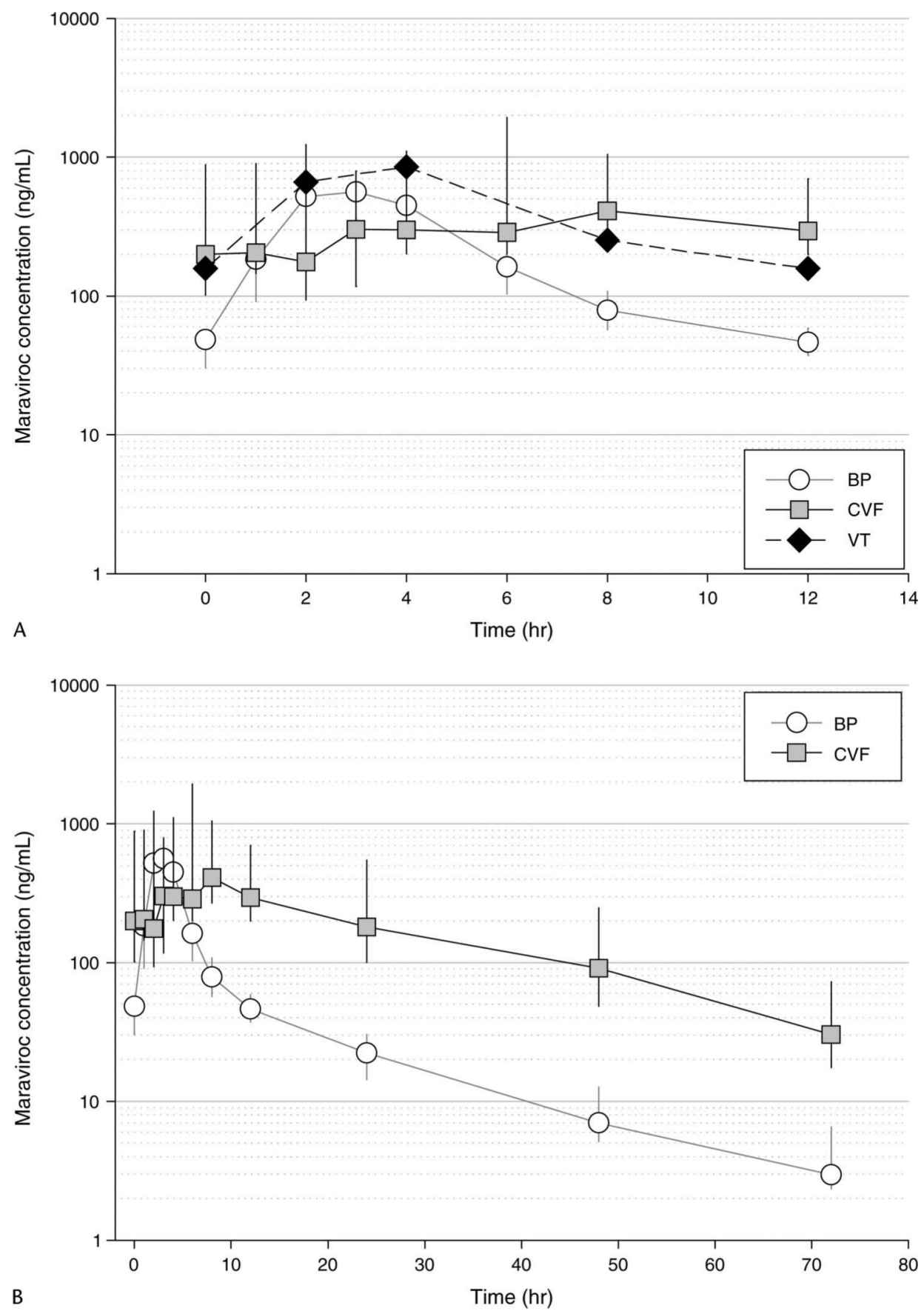

FIGURE 2.

$\mathrm{A}, \mathrm{VT}$ concentrations of maraviroc in relation to $\mathrm{BP}$ and CVF concentrations, within a dosing interval on day 7 (multiple dose) [data presented as median (IQR)]. An estimated tissue density of $1.05 \mathrm{~g} / \mathrm{mL}$ was used to convert VT concentration from nanograms per gram to nanograms per milliliter. B, Day 7 (multiple dose) concentration-time profile of maraviroc in BP and CVF over 72 hours post-dose for 10 subjects [data presented as median (IQR)]. IQR, interquartile range. 
TABLE 1

Demographics of Study Participants;Data Presented as Mean \pm SD or Number (\%)

\begin{tabular}{lc}
\hline Parameter & Result \\
\hline Age (yrs) & $26.9 \pm 5.4$ \\
Race & \\
African American & $4(33.3)$ \\
Asian & $1(8.3)$ \\
White & $6(50.0)$ \\
Mixed race & $1(8.3)$ \\
Weight $(\mathrm{kg})$ & $63.0 \pm 6.8$ \\
BMI $\left(\mathrm{kg} / \mathrm{m}^{2}\right)$ & $22.2 \pm 1.9$ \\
Oral contraceptives used during study & $9(75)$ \\
\hline
\end{tabular}

BMI, body mass index. 


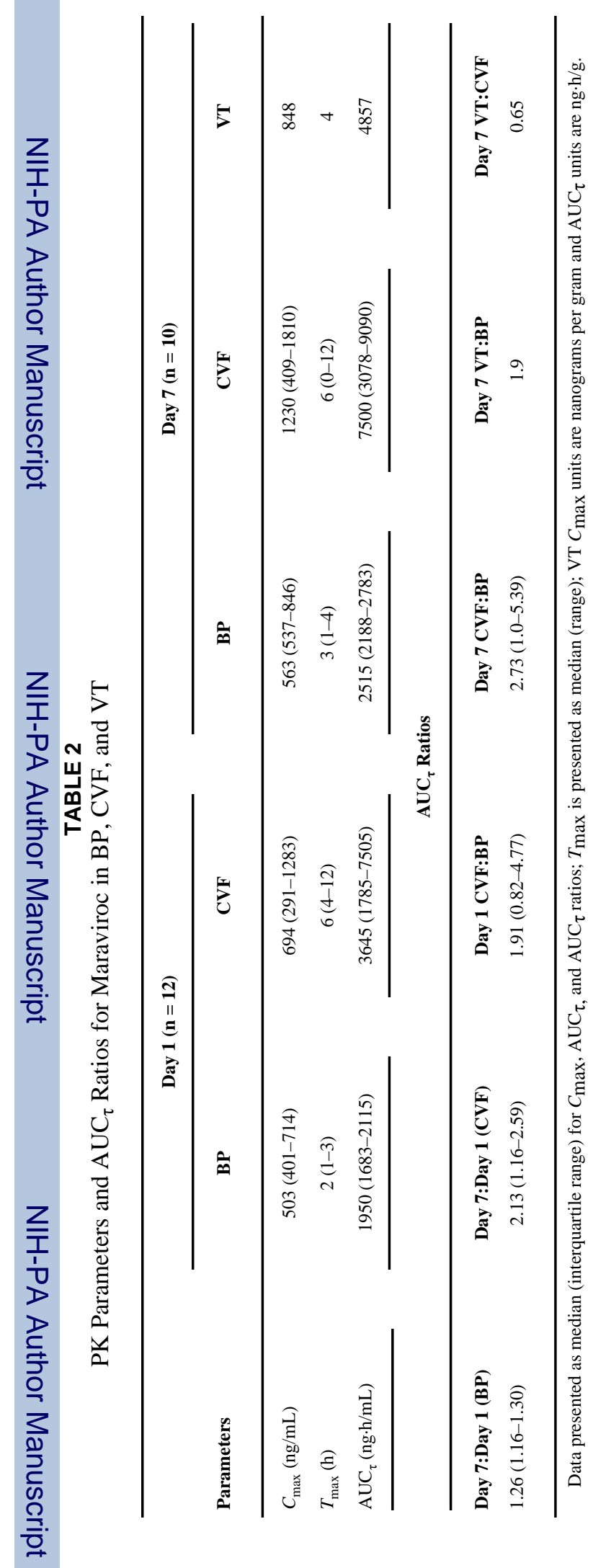

